\title{
Evaluation of Botanicals, Bio Agents and Fungicides against Stem Bleeding of Coconut caused by Thielviopsis paradoxa under in vitro Conditions
}

\author{
G.K. Sudarshan ${ }^{1 *}$, G.S. Chandrashekara ${ }^{1}$, T.B. Basavaraju ${ }^{1}$, \\ K.B. Palanna ${ }^{2}$ and G.P. Mutthuraju ${ }^{1}$ \\ ${ }^{1}$ University of Horticultural Sciences, Bagalkot-587104, Karnataka, India \\ ${ }^{2}$ Department of Plant Pathology, GKVK, Bangalore-560065, Karnataka, India \\ *Corresponding author
}

\begin{tabular}{|l}
\hline Key w o r d s \\
Coconut, \\
Fungicides, \\
Stem bleeding, \\
Thielviopsis \\
paradoxa, Plant \\
extracts, Bio agents
\end{tabular}

A B S T R A C T

Stem bleeding disease caused by Thielaviopsis paradoxa is one of the major diseases of coconut in almost all the coconut growing regions of Karnataaka. Bioefficacy of five botanicals viz. Allium sativa, Nerium olender, Tinospora cordifolia, Osimum sanctum and Aegle marmelos, Seven isolates namely Trichoderma viridae (GKVK), Trichoderma harzianum (GKVK), Trichoderma asperillum (GKVK), Trichoderma harzianum (HRS, NBAII, KRN and MYS) and ten systemic and three contact fungicides were evaluated under in vitro conditions against Thielviopsis paradoxa. Among the five botanicals evaluated only Allium sativa found significantly superior and showed complete inhibition of the growth of the pathogen at $15 \%$ and $20 \%$ concentration followed by Osimum sanctum found effective and has recorded eighty five per cent inhibition at $20 \%$ concentration. Among the antagonists tested Trichoderma viridae (GKVK) was found superior over all other bio agents by recording maximum inhibition of 78.00 per cent followed by Trichoderma asperillum $(G K V K)$ which has recorded 74.00 per cent. Among the thirteen fungicides evaluated standard check Carbendazim 50\%WP @ 0.1\%, Difenoconazole 25\% EC @ 0.1\%, Propiconazole 25\% EC@0.1\%, Tebuconazole 25.9\% EC@0.15\% and Thiophanate Methyl 70\%WP @ 0.28\% has recorded cent per cent inhibition and showed superior over other fungicides .The Azoxystrobin 23\%SC @ 0.1\% and Pencycuron 22.9\%SC @ 0.1\% were recorded least per cent inhibition on 9 DAI.

\section{Introduction}

Stem bleeding disease of coconut caused by Thielviopsis paradoxa (de seyness) von Hohnel is a debilitating disease and is widely prevalent in all coconut growing areas of the country. The disease was first reported from Sri Lanka (Petch, 1906) and later reported in India (Sundararaman, 1922) and other countries. In India, the disease is prevalent in almost all coconut growing states. The disease has been found to occur in all soil types, but more in laterite soils and sandy soils on seashore or backwater areas (Nambiar, 1994). The pathogen is a soil borne pathogen and enters the plant through growth cracks present on the stem and causes cortical decay. The disease is characterized by development of 
dark brown patches appearing at the basal portion of the trunk. A dark or reddish brown liquid exudates from the longitudinal cracks present on the stem bark. Subsequently, the liquid dries up and turns black; the tissues below the lesions become water soaked and get discolored. The lesion spread upwards as the disease progresses. In the advanced stages, stem gets tapered and crown size gets reduced leading to gradual reduction in yield. The earliest recommendation for the control of the disease was chiseling of the affected tissues and application of hot coal tar. This did not always results in giving effective control of the disease (Nambier and Kalpana sastry, 1988). Soil drenching with calixin 0.1\% (Radhakrishnan, 1990) and root feeding with Bavistin 5\% or Calixin 5\% (Ramanujam et al, 1993), have been found to reduce the disease to some extent. Biological control through the use of antagonistic microorganism and locally available botanicals for the development of integrated management strategy against the disease has emerged as available option (Alvindia and Natsuaki, 2008). Trichoderma spp., is considered to be antagonistic to many soil borne and plant pathogenic fungi (Prasad et al., 2002): Ramanujam et al., 2005 and Ranjana Chakrabarty et al., 2013). Soil application of Neem cake to the affected palms was found to reduce the population of T. paradoxa and encourage the antagonistic population in soil (Sanal Kumar et al., 1990). Locally available botanicals secrete antifungal metabolites that substantiate their action against certain fungi. IDM practices used to develop a combination of specific chemicals with bio-agents and botanicals with an aim to reduce the non-availability of bioagents and botanicals at specific period of time sand which are also environmental friendly. Since the pathogen is soil borne, it is essential to adopt an integrated approach involving antagonistic organisms, fungicides and acqueous plant extract for effective disease management. The present study was carried out to investigate the role of all three components in inhibiting the growth of the fungus in in vitro and subsequent formulation of IDM practices against the pathogen.

\section{Materials and Methods}

The study was carried out at Horticulture Research Station, Arsikere under All India coordinated research project. The fungus Thielviopsis paradoxa was isolated from diseased sample collected from stem bleeding affected coconut palm on potato dextrose agar. The six botanicals viz. Alliumsativa, Nerium olender, Tinospora cordifolia, Osimum sanctum, Aegle marmelos and Azadirecta indica were conducted at four concentrations by employing food poison technique (Nene, 1971). Fresh plant part materials were collected and washed first in tap water and then in distilled water. Aqueous plant extract was prepared by grinding $100 \mathrm{~g}$ fresh samples with $100 \mathrm{ml}$ distilled water (w/v) using a blender and filtrate was collected by passing through double layered muslin cloth. Finally filtrate thus obtained was used as stock solution. The seven bio control agents which were isolated from the soil samples of coconut rhizosphere from the different location i.e. Trichoderma viridae (GKVK), Trichoderma harzianum (GKVK), Trichoderma asperillum (GKVK), Trichoderma harzianum (HRS), Trichoderma harzianum (NBAII), Trichoderma harzianum (KRN) and Trichoderma harzianum (HRS) were evaluated by using dual culture method. Radial growth of Thielviopsis paradoxa was measured at different days after inoculation and the per cent inhibition was calculated. While ten systemic and three contact fungicides namely Azoxystrobin 23\%SC@0.1\%, Carbendazim50\%WP @ 0.1\%, Carboxin 37.5 + Thiram 37.5\%@DS @ 0.3\%, Copper oxy chloride 70\%WP @ $0.3 \%$, Difenoconazole 25\%EC@0.1\%, 
Pencycuron 22.9\% SC@0.1\%, Propiconazole 25\% EC@0.1\%, Tebuconazole 25.9\% EC @ $0.15 \%$, Tebuconazole + Trifloxystrobin 75\%WG@0.04\%, Tetraconazole 3.8\%w/w EW@0.1\%, Tetraconazole 3.8\%w/w EW@ $0.15 \%$, Thiophanate Methyl 70\%WP @ $0.28 \%$ and standard check Hexaconazole 5\% SC@0.1\% were evaluated at their recommended concentration using food poison technique. The radial growth of the test fungal colony recorded on $10^{\text {th }}$ day when maximum growth was observed in untreated control plates. The percent inhibition of the mycelia growth over control was calculated using formula given by Vincent (1942).

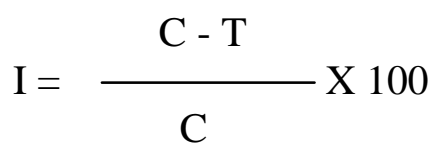

$\mathrm{I}=\%$ inhibition of mycelia growth

$\mathrm{C}=$ radial growth of fungus in control

$\mathrm{T}=$ radial growth of fungus in treatment

\section{Results and Discussion}

Influence of botanicals on mycelial growth of T. paradoxa was presented in Table 1 .

The results clearly reveal that among the six botanicals evaluated only Allium sativa found significantly superior and showed complete inhibition of the growth of the pathogen at $15 \%$ and $20 \%$ concentration followed by Osimum sanctum found effective in inhibiting the growth of Thielviopsis paradoxa at same concentration and they have recorded $65-79$ per cent inhibition (Table 1).

Seven bio control agents which were collected from the different locations were tested against Thielviopsis paradoxa by dual culture method. The results revealed that, all the antagonists significantly reduced the growth of Thielviopsis paradoxa by recording mycelial inhibition ranging from 61.33 to 78.00 per cent (Table 2).
Among the antagonists tested Trichoderma viridae $(G K V K)$ was found superior over all other bio agents by recording maximum inhibition of 78.00 per cent followed by Trichoderma asperillum (GKVK) which has recorded 74.00 per cent. Least inhibition of 61.33 per cent was recorded in Trichoderma harzianum (KRN).

Experiment on in vitro evaluation of ten systemic and three contact fungicides were carried out against Thielviopsis paradoxa (Table 3) and it was noticed that among the thirteen fungicides evaluated Among the thirteen fungicides evaluated standard check Carbendazim $\quad 50 \%$ WP @ $0.1 \%$, Difenoconazole $\quad 25 \% \quad$ EC@0.1\%, Propiconazole 25\% EC@0.1\%, Tebuconazole $25.9 \%$ EC@0.15\% and Thiophanate Methyl 70\%WP @ 0.28\% has recorded cent percent inhibition and showed superior over other fungicides .The Azoxystrobin 23\%SC@0.1\% and Pencycuron $22.9 \%$ SC @ $0.1 \%$ were recorded least per cent inhibition on 9 DAI.

The results in conformity with Ramanujam et al., 2005 and Ranjan Chakrabarty et al., 2013 where Allium sativum extract completely inhibited the growth of the pathogen.

Trichoderma viridae was found to be most effective with $61.62 \%$ inhibition followed by $T$. harzianum and $T$. virens with 60.80 and 59.49 per cent inhibition respectively, over control after $96 \mathrm{~h}$ of incubation period.

The fungicides, Calixin (Tridemorph 80EC) (a) $0.3 \%$ showed 100 per cent inhibition over control whereas Ridomil MZ-72, Blitox-50 and Bavistin showed 92.00, 91.55 and 91.44 per cent inhibition over control after $144 \mathrm{~h}$ of incubation respectively. 
Table.1 In vitro evaluation of different botanicals against stem bleeding of coconut caused by Thielviopsis paradoxa

\begin{tabular}{|c|c|c|c|c|c|c|c|c|c|c|c|c|c|c|}
\hline \multirow{4}{*}{$\begin{array}{l}\text { Trt } \\
\text { No. }\end{array}$} & \multirow[t]{4}{*}{ Treatment } & \multirow{4}{*}{$\begin{array}{l}\text { Part } \\
\text { used }\end{array}$} & \multicolumn{12}{|c|}{ \% Inhibition of Thielviopsis paradoxa } \\
\hline & & & \multicolumn{12}{|c|}{ Concentration } \\
\hline & & & \multicolumn{3}{|c|}{$5 \%$} & \multicolumn{3}{|c|}{$10 \%$} & \multicolumn{3}{|c|}{$15 \%$} & \multicolumn{3}{|c|}{$20 \%$} \\
\hline & & & 3DAI & 6DAI & 9DAI & 3DAI & 6DAI & 9DAI & 3DAI & 6DAI & 9DAI & 3DAI & 6DAI & 9DAI \\
\hline $\mathbf{T 1}$ & Allium sativa & Clove & $\begin{array}{c}77.63 \\
(61.77)\end{array}$ & $\begin{array}{c}70.94 \\
(57.40)\end{array}$ & $\begin{array}{c}73.33 \\
(58.91)\end{array}$ & $\begin{array}{c}80.48 \\
(63.78)\end{array}$ & $\begin{array}{c}79.81 \\
(63.30)\end{array}$ & $\begin{array}{c}81.56 \\
(64.58)\end{array}$ & $\begin{array}{l}100.00 \\
(90.00)\end{array}$ & $\begin{array}{l}100.00 \\
(90.00)\end{array}$ & $\begin{array}{l}100.00 \\
(90.00)\end{array}$ & $\begin{array}{l}100.00 \\
(90.00)\end{array}$ & $\begin{array}{l}100.00 \\
(90.00)\end{array}$ & $\begin{array}{l}100.00 \\
(90.00)\end{array}$ \\
\hline $\mathbf{T} 2$ & $\begin{array}{l}\text { Tinospora } \\
\text { cordifolia }\end{array}$ & Leaf & $\begin{array}{c}2.85 \\
(8.58)\end{array}$ & $\begin{array}{c}5.77 \\
(13.77)\end{array}$ & $\begin{array}{c}0.00 \\
(0.00)\end{array}$ & $\begin{array}{c}1.90 \\
(7.10)\end{array}$ & $\begin{array}{c}18.19 \\
(25.75)\end{array}$ & $\begin{array}{c}18.44 \\
(25.43)\end{array}$ & $\begin{array}{c}0.49 \\
(1.80)\end{array}$ & $\begin{array}{c}20.48 \\
(26.90)\end{array}$ & $\begin{array}{c}29.78 \\
(33.05)\end{array}$ & $\begin{array}{c}2.94 \\
(8.72)\end{array}$ & $\begin{array}{c}21.13 \\
(27.29)\end{array}$ & $\begin{array}{c}0.00 \\
(0.00)\end{array}$ \\
\hline $\mathbf{T 3}$ & Nerium olender & Leaf & $\begin{array}{c}3.33 \\
(10.37)\end{array}$ & $\begin{array}{c}6.75 \\
(14.68)\end{array}$ & $\begin{array}{c}1.56 \\
(4.44)\end{array}$ & $\begin{array}{c}24.29 \\
(29.51)\end{array}$ & $\begin{array}{c}21.43 \\
(27.57)\end{array}$ & $\begin{array}{c}19.78 \\
(26.39)\end{array}$ & $\begin{array}{c}27.10 \\
(31.35)\end{array}$ & $\begin{array}{c}23.27 \\
(28.83)\end{array}$ & $\begin{array}{c}10.67 \\
(19.06)\end{array}$ & $\begin{array}{c}27.57 \\
(31.64)\end{array}$ & $\begin{array}{c}25.14 \\
(30.06)\end{array}$ & $\begin{array}{c}21.42 \\
(19.82)\end{array}$ \\
\hline T4 & Osimum sanctum & Leaf & $\begin{array}{c}48.08 \\
(43.90)\end{array}$ & $\begin{array}{c}33.71 \\
(35.46)\end{array}$ & $\begin{array}{c}4.22 \\
(11.74)\end{array}$ & $\begin{array}{c}58.10 \\
(49.67)\end{array}$ & $\begin{array}{c}57.87 \\
(49.53)\end{array}$ & $\begin{array}{c}49.11 \\
(44.49)\end{array}$ & $\begin{array}{c}74.88 \\
(59.92)\end{array}$ & $\begin{array}{c}75.47 \\
(60.32)\end{array}$ & $\begin{array}{c}76.00 \\
(60.67)\end{array}$ & $\begin{array}{c}77.34 \\
(61.58)\end{array}$ & $\begin{array}{c}77.90 \\
(61.97)\end{array}$ & $\begin{array}{c}85.06 \\
(63.92)\end{array}$ \\
\hline T5 & Aegle marmelos & Leaf & $\begin{array}{c}3.80 \\
(11.10)\end{array}$ & $\begin{array}{c}12.54 \\
(20.51)\end{array}$ & $\begin{array}{c}0.00 \\
(0.00)\end{array}$ & $\begin{array}{c}5.24 \\
(10.89)\end{array}$ & $\begin{array}{c}25.43 \\
(30.26)\end{array}$ & $\begin{array}{c}11.78 \\
(20.02)\end{array}$ & $\begin{array}{c}13.28 \\
(21.32)\end{array}$ & $\begin{array}{c}24.49 \\
(29.64)\end{array}$ & $\begin{array}{c}1.78 \\
(7.45)\end{array}$ & $\begin{array}{c}13.79 \\
(21.76)\end{array}$ & $\begin{array}{c}23.00 \\
(28.65)\end{array}$ & $\begin{array}{c}18.43 \\
(18.84)\end{array}$ \\
\hline T6 & Azadirecta indica & Leaf & $\begin{array}{c}48.09 \\
(43.91)\end{array}$ & $\begin{array}{c}39.56 \\
(38.97)\end{array}$ & $\begin{array}{c}11.33 \\
(19.62)\end{array}$ & $\begin{array}{c}49.05 \\
(44.45)\end{array}$ & $\begin{array}{c}43.74 \\
(41.40)\end{array}$ & $\begin{array}{c}12.89 \\
(12.22)\end{array}$ & $\begin{array}{c}59.10 \\
(50.25)\end{array}$ & $\begin{array}{c}66.45 \\
(54.61)\end{array}$ & $\begin{array}{c}65.56 \\
(54.07)\end{array}$ & $\begin{array}{c}69.43 \\
(56.46)\end{array}$ & $\begin{array}{c}66.86 \\
(54.86)\end{array}$ & $\begin{array}{c}60.37 \\
(60.07)\end{array}$ \\
\hline & SEm \pm & & 1.17 & 1.36 & 1.28 & 1.82 & 0.51 & 0.56 & 0.91 & 0.37 & 0.57 & 1.10 & 0.60 & 0.36 \\
\hline & CD@ 1\% & & 4.70 & 5.47 & 5.13 & 7.30 & 2.03 & 2.24 & 3.66 & 1.49 & 2.28 & 4.40 & 2.43 & 1.45 \\
\hline
\end{tabular}


Table. 2 In vitro evaluation of Bio control agents against stem bleeding of coconut caused by Thielviopsis paradoxa

\begin{tabular}{|c|c|c|c|c|}
\hline \multirow{2}{*}{$\begin{array}{c}\text { Treatment } \\
\text { No. }\end{array}$} & Bio control agents & \multicolumn{3}{|c|}{ \% Inhibition of Thielviopsis paradoxa } \\
\hline T1 & Trichoderma viridae $(G K V K)$ & $3 \mathrm{DAI}$ & $6 \mathrm{DAI}$ & 9DAI \\
\hline & & 39.28 & 73.96 & 78.00 \\
& & $(38.79)$ & $(59.33)$ & $(62.03)$ \\
\hline T2 & Trichoderma harzianum $(G K V K)$ & 24.12 & 73.67 & 64.67 \\
& & $(29.24)$ & $(59.14)$ & $(53.53)$ \\
\hline T3 & Trichoderma asperillum $(G K V K)$ & 22.16 & 70.51 & 74.00 \\
& & $(28.00)$ & $(57.11)$ & $(59.36)$ \\
\hline T4 & Trichoderma harzianum $(H R S)$ & 23.16 & 75.47 & 72.89 \\
& & $(28.69)$ & $(60.32)$ & $(58.62)$ \\
\hline T5 & Trichoderma harzianum $(N B A I I)$ & 22.21 & 61.97 & 67.56 \\
& & $(27.95)$ & $(51.94)$ & $(55.28)$ \\
\hline T6 & Trichoderma harzianum $($ KRN) & 19.21 & 58.19 & 61.33 \\
& & $(25.82)$ & $(49.72)$ & $(51.55)$ \\
\hline T7 & Trichoderma harzianum $(M Y S)$ & 23.98 & 64.05 & 70.22 \\
& & $(29.29)$ & $(53.16)$ & $(56.96)$ \\
\hline & SEm \pm & 0.73 & 0.33 & 0.43 \\
\hline & CD $(\mathrm{P}=0.01)$ & 11.56 & 2.93 & 3.83 \\
\hline
\end{tabular}

Table.3 In vitro evaluation of fungicides against stem bleeding of coconut caused by Thielviopsis paradoxa

\begin{tabular}{|c|c|c|c|c|c|}
\hline \multirow{2}{*}{$\begin{array}{c}\text { Treatment } \\
\text { No. }\end{array}$} & \multirow[t]{2}{*}{ Fungicide } & \multirow[t]{2}{*}{ Dosage (\%) } & \multicolumn{3}{|c|}{ \% Inhibition of T. paradoxa } \\
\hline & & & 3DAI & 6DAI & 9DAI \\
\hline T1 & Azosystrobin $23 \% \mathrm{SC}$ & 0.1 & $\begin{array}{c}35.73 \\
(36.60)\end{array}$ & $\begin{array}{c}4.00 \\
(8.98)\end{array}$ & $\begin{array}{c}1.56 \\
(6.21)\end{array}$ \\
\hline $\mathbf{T 2}$ & Carbendazim50\%WP & 0.1 & $\begin{array}{l}100.00 \\
(90.00)\end{array}$ & $\begin{array}{l}100.00 \\
(90.00)\end{array}$ & $\begin{array}{l}100.00 \\
(90.00)\end{array}$ \\
\hline T3 & Carboxin 37.5 + Thiram 37.5\%DS & 0.3 & $\begin{array}{l}100.00 \\
(90.00)\end{array}$ & $\begin{array}{l}100.00 \\
(90.00)\end{array}$ & $\begin{array}{c}85.37 \\
(67.53)\end{array}$ \\
\hline T4 & Copper oxy chloride $50 \% \mathrm{WP}$ & 0.3 & $\begin{array}{l}100.00 \\
(90.00)\end{array}$ & $\begin{array}{l}100.00 \\
(90.00)\end{array}$ & $\begin{array}{l}100.00 \\
(90.00)\end{array}$ \\
\hline T5 & Difenoconazole $25 \% \mathrm{EC}$ & 0.1 & $\begin{array}{r}100.00 \\
(90.00)\end{array}$ & $\begin{array}{l}100.00 \\
(90.00)\end{array}$ & $\begin{array}{l}100.00 \\
(90.00)\end{array}$ \\
\hline T6 & Hexaconazole $5 \% \mathrm{SC}$ & 0.1 & $\begin{array}{l}100.00 \\
(90.00)\end{array}$ & $\begin{array}{c}78.13 \\
(62.13)\end{array}$ & $\begin{array}{c}67.37 \\
(55.17)\end{array}$ \\
\hline T7 & Pencycuron $22.9 \%$ SC & 0.1 & $\begin{array}{c}32.80 \\
(34.93)\end{array}$ & $\begin{array}{c}0.00 \\
(0.00)\end{array}$ & $\begin{array}{c}1.82 \\
(7.52)\end{array}$ \\
\hline T8 & Propiconazole $25 \% \mathrm{EC}$ & 0.1 & $\begin{array}{l}100.00 \\
(90.00)\end{array}$ & $\begin{array}{l}100.00 \\
(90.00)\end{array}$ & $\begin{array}{l}100.00 \\
(90.00)\end{array}$ \\
\hline T9 & Tebuconazole $25.9 \% \mathrm{EC}$ & 0.15 & $\begin{array}{l}100.00 \\
(90.00)\end{array}$ & $\begin{array}{l}100.00 \\
(90.00)\end{array}$ & $\begin{array}{l}100.00 \\
(90.00)\end{array}$ \\
\hline T10 & Tebuconazole+ Trifloxystrobin75\%WG & 0.04 & $\begin{array}{c}77.76 \\
(61.89)\end{array}$ & $\begin{array}{c}82.13 \\
(65.00)\end{array}$ & $\begin{array}{c}81.18 \\
(64.32)\end{array}$ \\
\hline T11 & Thiophanate Methyl 70\%WP & 0.28 & $\begin{array}{l}100.00 \\
(90.00)\end{array}$ & $\begin{array}{l}100.00 \\
(90.00)\end{array}$ & $\begin{array}{l}100.00 \\
(90.00)\end{array}$ \\
\hline T12 & Tetraconazole $3.8 \% \mathrm{w} / \mathrm{w}(4 \% \mathrm{w} / \mathrm{v}) \mathrm{EW}$ & 0.1 & $\begin{array}{l}100.00 \\
(90.00)\end{array}$ & $\begin{array}{c}74.40 \\
(59.61)\end{array}$ & $\begin{array}{c}63.46 \\
(52.81)\end{array}$ \\
\hline \multirow[t]{3}{*}{ T13 } & Tetraconazole $3.8 \% \mathrm{w} / \mathrm{w}(4 \% \mathrm{w} / \mathrm{v}) \mathrm{EW}$ & 0.15 & $\begin{array}{l}100.00 \\
(90.00)\end{array}$ & $\begin{array}{c}76.00 \\
(60.69)\end{array}$ & $\begin{array}{c}67.10 \\
(55.01)\end{array}$ \\
\hline & \multicolumn{2}{|l|}{$\mathrm{SEm} \pm$} & 0.67 & 1.07 & 0.63 \\
\hline & \multicolumn{2}{|l|}{$\mathrm{CD}(\mathrm{P}=0.01)$} & 5.08 & 8.18 & 4.77 \\
\hline
\end{tabular}




\section{References}

Alvindia, D. G. and Natsuaki, K. T. 2009. Biocontrol activities of Bacillus amylolifaciens DGA 14 isolated from banana fruit surface against banana crown rot- causing pathogens. Crop Prot. 28: 236- 242.

Nambiar, K.K.N.1994. Diseases and disorders of coconuts. In: Advances in Horticulture vol. 10 Plantation and Spice Crops Part 2. K.L.Chadha and P. Rethinam, (Eds.). Malhotra Publishing House, New Delhi., pp-857-882.

Nambiar, K. K. N. and Kalpana Sastry, R. 1988. Stem bleeding disease of coconut: Current status and Approaches for its control. Philippine J. Coconut studies. 12(9): 13-14.

Nene, Y. L. 1971. Fungicides in plant disease control. Oxford and IBH Publ. Co. New Delhi., pp. 386.

Petch, T. 1906, Diseases of the coconut palm. Trop. Agriculturist. 27:490-491.

Prasad, R. D., Rangeshwaran, R., Hedge, S. V. and Anuroop, C. P. 2002. Effect of soil application of Trichoderma harzianum on pigeon pea wilt caused by Fusarium udum under field conditions. Crop Prot. 21: 293-297.

Radhakrishnan, T.C.1990, Control of stem bleeding disease of coconut. Indian Coconut J. 20(9):13-14.

Ramanujam, B., Nambiar, K.K.N. and Anil Kumar. 1993. Chemical control of stem bleeding disease of coconut. In: Advances in coconut Research and Development, (Eds.) M.K. Nair, H.H.
Khan, P. Gopalasundaram and E.V.V.B. Rao Oxford and IBH publishing Co. Pvt. Ltd., New Delhi., pp- 615-616.

Ramanujam, B., Nambiar, K. K. N and Iyer, R. 2005. Effect of systemic fungicides, aqueous extracts of oil cake and inorganic soil amendment on Thielaviopsis paradoxa and its antagonistic fungi in vitro. $J$. Plantation Crops. 33(2): 107-111.

Ranjana chakrabarty, G.C.Acharya and T.C.Sarma, 2013, Effect of Fungicides, Trichoderma and Plant extracts on mycelial growth of Thielviopsis paradoxa, under in vitro condition. The Bioscan .8(1): 55-58.

Ranjana Chakrabartty, G.C. Acharya and T.C. Sarma. 2013. Management of Basal stem rot of Areca nut (Areca catechu L.) under Assam condition. The Biosean.8 (4):1291-1294.

Sanal kumar, R., Rohini Iyer and Nambiar, K.K.N, 193, In vitro inhibition of Thielviopsis paradoxa by mycoflora from coconut basin. In advances in coconut Research and Development, (Eds.) M.K. Nair, H.H. Khan, P. Gopalasundaram and E.V.V.B. Rao Oxford and IBH publishing Co. Pvt. Ltd., New Delhi. pp 613-614.

Sundraraman, S. 1922. The coconut bleeding disease. Pusa Agr. Res. Inst. Bull. 127: $8 p$.

Vincent, J.M.1947. Distortion of fungal hyphae in the presence of certain inhibitors. Nature, 159:850

\section{How to cite this article:}

Sudarshan, G.K., G.S. Chandrashekara, T.B. Basavaraju, K.B. Palanna and G.P. Mutthuraju. 2019. Evaluation of Botanicals, Bio Agents and Fungicides against Stem Bleeding of Coconut caused by Thielviopsis Paradoxa under Invitro Conditions. Int.J.Curr.Microbiol.App.Sci. 8(02): 2690-2695. doi: https://doi.org/10.20546/ijcmas.2019.802.314 